J. E. Bertie: The general answer is that, because the structures differ, there are twice as many branches of dispersion curves for ice Ih as for ice Ic, so differences between the spectra of the two phases are to be expected. A more specific answer depends on the model you assume for the force field appropriate to the translational vibration. If you use the force field for ice Ih that Klug, Wong, and Whalley presented at Ottawa in 1972, the splitting is explained by two branches of dispersion curves which are fairly flat across the Brillouin zone near $\mathrm{I} 6 \mathrm{c} \mathrm{cm}^{-1}$, and which yield two peaks in the density of vibrational states according to the histogram that Klug and others reported. With other force fields the explanation may differ in detail but must be the same in principle.

J. W. Glen: Is there any evidence that the differences found for the hydrate of cyclopropane as compared to ethylene oxide and trimethylene oxide correspond to any ordering of protons in the cage or to modifications of the cage electrical properties (presumably caused by electrical interaction with the guest molecule)?

Bertie: There is no evidence at all to suggest that the protons in the water lattice order at any temperature for any clathrate hydrate of structure I or II. Hexamethylene tetramine hydrate does contain partially ordered protons, but it has a quite different structure, in which the nitrogen atoms are hydrogen-bonded to the water molecules. The dielectric relaxation of the water molecules is much faster, at a given temperature, for the hydrates of the dipolar guest molecules than for those of cyclopropane. This may well be due to the interaction of the dipole moment of the guest molecule with the water molecules, in which case it has the same origin, we believe, as has the difference between the $\nu_{\mathrm{OD}}(\mathrm{HDO})$ bands of cyclopropane hydrate and those of the dipolar guest molecules.

\title{
DIELECTRIC BEHAVIOUR OF ICE MICROCRYSTALS: A STUDY VERSUS TEMPERATURE
}

\author{
By Christian Boned, Bernard Lagourette and Marc Clausse
}

(Université de Pau et des Pays de l'Adour, Institut Universitaire de Recherche Scientifique, Laboratoire de Thermodynamique, Avenue Philippon, F-640oo Pau, France)

Abstract. Dispersions of ice microcrystals were obtained from the breakdown of supercooling of water-in-oil type emulsions whose disperse phase was either resin-exchanged water or aqueous solutions of $\mathrm{NH}_{4} \mathrm{Cl}$. Their complex permittivity $\epsilon^{\star}=\epsilon^{\prime}-j \epsilon^{\prime \prime}$ was studied versus their temperature $T$, up to the melting point of the ice microcrystals, by means of an automatically balancing admittance bridge (General Radio I $68 \mathrm{oA}$ ) working at $400 \mathrm{~Hz}$ and I kHz. The plots $\epsilon^{\prime}(T), \epsilon^{\prime \prime}(T)$, and $\epsilon^{\prime \prime}\left(\epsilon^{\prime}\right)$ reveal that these systems exhibit two distinct dielectric relaxations located on both sides of a temperature $T_{m}$ which was found to be equal to about $-20^{\circ} \mathrm{C}$ in the case of water and lower than $-20^{\circ} \mathrm{C}$ in the case of aqueous solutions of $\mathrm{NH}_{4} \mathrm{Cl}$. The relaxation located in the lower temperature range arises from the Debye dipolar absorption of ice while the other one could be related to structural changes occurring within the 
lattice of ice as $T$ approaches its melting point. These results are consistent with those obtained by investigating versus frequency the dielectric behaviour of ice microcrystals, at discrete sub-zero temperatures close to their melting point.

This paper has been accepted for publication in full in a forthcoming issue of the fournal of Glaciology.

\section{DISCUSSION}

J. G. Paren: You have pointed out one failure of the Hanai model, i.e. $\epsilon_{1} \neq \epsilon_{2} /(\mathrm{I}-\phi)^{3}$ at the lowest frequencies. Can you confirm what part of the emulsion response can be easily interpreted quantitatively by a mixture model?

C. Boned: A study has been made in our laboratory concerning the dielectric behaviour of emulsions, especially of the water-in-oil type. It has been shown (Clausse, unpublished; Clausse and Royer, I 976) that the dielectric features of emulsions can be correctly represented by using Hanai's law and its derivative formulae. A first attempt was made in 197 I (Clausse, unpublished) to apply Hanai's formula to the study of the dielectric behaviour of ice microcrystal dispersions. This attempt proved that Hanai's formula cannot be applied to dispersions of ice if it is assumed that ice microcrystals exhibit merely a bulk conduction in addition to Debye dipolar absorption. A more sophisticated model has been designed by Lagourette and others $(1976)$ to interpret the results obtained on stabilized ice dispersions through variablefrequency measurements. They assumed that disordered essentially conductive clusters exist within the ice lattice. On this basis, the dielectric properties of stabilized ice dispersions can be described by using an appropriate application of Hanai's formula. As concerns the variabletemperature study that has been presented here, the same procedure can be followed. So one cannot speak of a failure of Hanai's formula.

K. Itagaki: I have observed various anomalous features such as negative conductance in attempts to measure the dielectric behaviour of single-crystal ice by filling the space between electrodes and ice samples with oils of various dielectric constants. Is your equipment capable of handling anomalies?

Boned: We never had the opportunity to observe anomalous dielectric features such as negative conductance. We would suggest that such phenomena may be artefacts arising from inductance effects or from a static electrification of the insulating oil. The General Radio I68oA automatically balancing bridge is a versatile instrument that can give either the capacitance and conductance or the capacitance and loss angle. Consequently, inductance effects could be detected when using it.

\section{REFERENCES}

Clausse, M. Unpublished. Contribution à l'étude des propriétés diélectriques des émulsions. [Dr. ès. Sc. thesis, Université de Pau et des Pays de l'Adour, r972.]

Clausse, M., and Royer, R. 1976. Computerized treatment of Hanai's formula, and its application to the study of the dielectric behavior of emulsions. (In Kerker, M., ed. Colloid and interface science. Vol. 2. Aerosols, emulsions, and surfactants. Proceedings of the International Conference on Colloids and Surfaces-5oth Colloid and Surface Science Symposium, held in San Juan, Puerto Rico, on June 21-25, 1976. New York, etc., Academic Press Inc., p. $217-32$.)

Lagourette, B., and others. 1976. Study of the dielectric properties of disperse micro-crystals of ice near the melting temperature. Section II: discussion and interpretation, [par] B. Lagourette, C. Boned et R. Royer. Journal de Physique, Tom. 37, Nos. 7-8, p. $955^{-64}$. 